\title{
Structural Studies on the Phosphorus-Nitrogen Bond. I. The Crystal Structure of Tris(morpholino)phosphine and Tris(piperidino)phosphine
}

\author{
CHRISTIAN RØMMING ${ }^{\mathrm{a}}$ and JON SONGSTAD ${ }^{\mathrm{b}}$
}

${ }^{\mathrm{a}}$ Department of Chemistry, University of Oslo, Oslo 3, Norway and ${ }^{\mathrm{b}}$ Department of Chemistry, University of Bergen, 5014 Bergen-Univ., Norway

The structures of the title compounds $\left[\mathrm{O}\left(\mathrm{CH}_{2} \mathrm{CH}_{2}\right)_{2} \mathrm{~N}\right]_{3} \mathrm{P}, \mathrm{I}$, and $\left[\mathrm{CH}_{2}\left(\mathrm{CH}_{2} \mathrm{CH}_{2}\right)_{2} \mathrm{~N}\right]_{3} \mathrm{P}$, II, have been determined by $\mathrm{X}$-ray diffraction techniques. I is triclinic, space group $P \overline{1}$, with $a=8.734(3)$ $\AA, b=9.299(2) \AA, c=11.357(3) \AA ; \alpha=73.91(2)^{\circ}$, $\beta=83.29(2)^{\circ}$ and $\gamma=117.88(2)^{\circ}, Z=2$. II is monoclinic, space group $P 2_{1} / c$, with (at $-150{ }^{\circ} \mathrm{C}$ ) $a=8.931(4) \AA, b=23.507(6) \AA, c=15.428(4) \AA$ and $\beta=90.44(3)^{\circ}, Z=8$. Full-matrix least-squares refinement led to a final $R$-value of 0.062 for 2056 reflections for I, and 0.072 for 5218 reflections for II.

The two compounds exhibit several similar structural features: (i) Two small NPN bond angles of $98^{\circ}$ and one large of $110^{\circ}$; (ii) two short $\mathrm{P}-\mathrm{N}$ bonds between 1.69 and $1.70 \AA$ while the third is longer, $1.726 \AA$; (iii) one of the nitrogen atoms, the one linked through the long bond to the phosphorus atom, is essentially $s p^{3}$ hybridized and its lone pair is anti to the phosphorus lone pair; (iv) the remaining two nitrogen atoms are mainly $s p^{2}$ hybridized and their lone pairs are roughly normal to the phosphorus lone pair and also to each other. The main difference between the two molecules is that in I two of the morpholino groups are twisted in opposite directions whereas in II two piperidino groups are twisted in the same direction.

A correlation between the sums of the bond angles around the nitrogen atoms and the corresponding $\mathrm{P}-\mathrm{N}$ bond lengths has been observed.

In recent years there has been a controversy with regard to the structure of tris(dialkylamino)phosphines, $\left(\mathrm{R}_{2} \mathrm{~N}\right)_{3} \mathrm{P}^{1-4}$ The main subject for the dispute has been the direction of the nitrogen lone pairs relative to that of the phosphorus lone pair and whether these compounds possess local $C_{3 v}$ or $C_{s}$ symmetry (for a detailed review, $c f$. Ref. 5). Some of the possible models suggested so far are shown in Fig. 1. Several other models are just as conceivable, e.g. those having the nitrogen lone pairs skew to the phosphorus lone pair in a propeller-like arrangement. ${ }^{4}$ It should be emphasized, however, that the structural models considered for this class of tervalent phosphorus compounds are based upon the assumption that the nitrogen atoms are $s p^{2}$ hybridized. An X-ray study of difluorodimethylaminophosphine, $\mathrm{Me}_{2} \mathrm{NPF}_{2}{ }^{6}$, has revealed that the nitrogen atom and its neighbours are coplanar. Similar conclusions have been arrived at from electron diffraction studies of tris(dimethylamino)phosphine, $\left(\mathrm{Me}_{2} \mathrm{~N}\right)_{3} \mathrm{P},{ }^{7}$ and from microwave studies of related compounds. ${ }^{8,9}$

Recently, from studies of tris(dialkylamino)phosphines by photoelectron spectroscopy, the
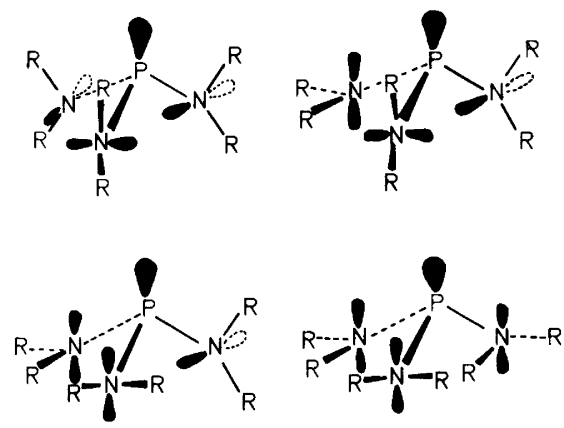

Fig. 1. Models suggested for the lone pair directions in tris(dialkylamino)phosphines. 
accuracy and thus the structural conclusions from the electron-diffraction experiments have been seriously critisized. ${ }^{1,4,5}$ Furthermore, the occurrence of nitrogen atoms with coplanar bonds may not be as general as has been believed; $\mathrm{Me}_{2} \mathrm{NPF}_{2}$, for example, does not have a planar nitrogen configuration in the gas phase. ${ }^{10}$ It is also difficult to understand how tris(dialkylamino)phosphines can act as powerful nitrogen nucleophiles toward a number of substrates ${ }^{11,12}$ when the nitrogen atoms have a planar structure.

Finally, the problem with regard to the bond order of the phosphorus-nitrogen bond arises. ${ }^{13}$ Two opposing views have been forwarded as to the direction of the charge transfer in this type of bond; the $p_{\mathrm{N}} \rightarrow d_{\mathrm{P}}$ transfer mechanism has been preferred by some authors, ${ }^{14-17}$ whereas others find evidence for the reverse transfer without the use of $d$-orbitals, that is, electron transfer from the phosphorus atom to the more electronegative nitrogen atom. ${ }^{18-23}$

In an attempt to solve some of the many problems with regard to both structure and bonding in aminophosphines and related compounds we have started a structural investigation of this class of compounds. In the present paper the X-ray crystal structure determinations of tris(morpholino)phosphine, I, and tris(piperidino)phosphine, II, are reported. Since the steric demands of the substituents in the two compounds would be expected to be comparable to those of other dialkylamino groups, the structures of I and II may be typical for

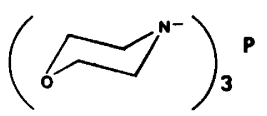

I

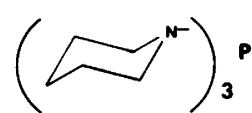

II the structure of $\left(\mathrm{R}_{2} \mathrm{~N}\right)_{3} \mathrm{P}(\mathrm{R}=\mathrm{Me}, \mathrm{Et}, \mathrm{Pr}$ etc. $)$. The latter compounds are not readily attacked by $\mathrm{X}$-ray methods since they are liquids, even at low temperatures, whereas I and II both are crystalline at room temperatures and stable provided atmospheric moisture is excluded.

\section{EXPERIMENTAL}

Materials. Tris(morpholino)phosphine, I, was prepared from phosphorus trichloride and morpholine as previously described. ${ }^{24}$ The product was repeatedly crystallized from toluene to remove traces of morpholine hydrochloride. Suitable crystals were grown from acetonitrile; the specimen used for the X-ray experiments was cut to approximate dimensions $0.3 \times 0.3 \times 0.1 \mathrm{~mm}$. Tris(piperidino)phosphine, II, was made in a similar way but in diethyl ether as solvent. After filtration and removal of the solvent the oily product was dissolved in a large volume of diethyl ether and kept for several days at $-20{ }^{\circ} \mathrm{C}$ to allow traces of piperidine hydrochloride to precipitate. A crystalline product separated from a fairly concentrated etheral solution at dry ice temperature. Crystals suitable for the $\mathrm{X}$-ray study were obtained by sublimation; the specimen used had approximate dimensions $0.2 \times 0.2 \times 0.2 \mathrm{~mm}$.

$X$-Ray data. Data for the measurements of cell dimensions and intensity data were obtained on a SYNTEX $P \overline{1}$ diffractometer at $18 \pm 1{ }^{\circ} \mathrm{C}$ (I) and $-150{ }^{\circ} \mathrm{C}(\mathrm{II})$, respectively, using graphite crystal monochromated MoK $\alpha$ radiation $(\lambda=0.71069 \AA)$. Cell parameters were determined by a least-squares fit to the diffractometer settings for 15 general reflections with $20^{\circ}<2 \theta<30^{\circ}$. Intensities were collected with the $\theta-2 \theta$ scan technique, scan speed $4^{\circ} \min ^{-1}$ for compound I and 3-6 ${ }^{\circ} \mathrm{min}^{-1}$ depending on the peak intensity for compound II, scan width $\pm 1.2^{\circ}$ (I) and $\pm 0.6^{\circ}$ (II), up to a $\sin \theta / \lambda$ value of $0.60 \AA^{-1}$ for I and $0.70 \AA^{-1}$ for II. Background counts were taken for 0.35 times the scan time at each of the scan limits. Three standard reflections were measured at regular intervals during the data collection; variations less than $2.5 \%$ were observed for I, whereas a decrease of $15 \%$ in the intensity of the standard reflections was observed for II; the intensities were adjusted according to this drift. Out of the 2639 unique reflections recorded from I, 2056 with $I>2.5 \sigma(I)$ were retained for the structure analysis; the corresponding numbers for II were 6203 and 5218. The standard deviations for the intensities were calculated by $\sigma(I)=\left[C_{\mathrm{T}}+\left(0.03 C_{\mathrm{N}}\right)^{2}\right]^{\frac{1}{2}}$, where $C_{\mathrm{T}}$ is the total number of counts and $C_{\mathrm{N}}$ is the scan count minus background count. The intensities were corrected for Lorentz and polarization effects but not for absorption.

Description of the computer programs applied for the structure analyses is given in Ref. 25 . In the full-matrix least-squares program the quantity minimized was $\Sigma w \Delta F^{2}$ where $w$ is the inverse of the variance of the observed structure factors; for reflections with $\sin \theta / \lambda<0.45 \AA^{-1} w$ was multiplied with a function of $\sin \theta / \hat{\lambda}$ to give less weight to low-order reflections. Atomic form factors were those of Doyle and Turner ${ }^{26}$ for $\mathrm{P}, \mathrm{O}, \mathrm{N}$ and $\mathrm{C}$ and of Stewart, Davidson and Simpson ${ }^{27}$ for $\mathrm{H}$. 


\section{CRYSTAL DATA}

I. Tris(morpholino)phosphine, $\mathrm{C}_{12} \mathrm{H}_{24} \mathrm{O}_{3} \mathrm{~N}_{3} \mathrm{P}$, m.p. $157^{\circ} \mathrm{C}$. Triclinic, $a=8.734(3) \AA ; b=9.299(2) \AA$; $c=11.357(3) \AA ; \alpha=73.91(2)^{\circ} ; \beta=83.29(2)^{\circ} ; y=$ $117.88(2)^{\circ} ; V=749.6 \AA^{3} ;\left(t=18 \pm 1{ }^{\circ} \mathrm{C}\right) ; M=289.31$; $Z=2 ; \quad F \quad(000)=312 ; \mu(\operatorname{MoK} \alpha)=2.2 \mathrm{~cm}^{-1} ; \quad D_{m}$ (flotation) $=1.27 \mathrm{~g} \mathrm{~cm}^{-3} ; D_{x}=1.282 \mathrm{~g} \mathrm{~cm}^{-3}$. Space group $P \overline{1}$ (No. 2).

II. Tris(piperidino)phosphine, $\mathrm{C}_{15} \mathrm{H}_{30} \mathrm{~N}_{3} \mathrm{P}$, m.p. $37{ }^{\circ} \mathrm{C}$. Monoclinic, $a=8.931(4) \AA ; b=23.507(6) \AA$; $c=15.428(4) \quad \AA ; \quad \beta=90.44(3)^{\circ} ; \quad V=3238.8 \quad \AA^{3} ;$ $\left(t=-150{ }^{\circ} \mathrm{C}\right) ; M=283.40 ; Z=8 ; F(000)=1248$; $\mu(\mathrm{MoK} \alpha)=1.0 \mathrm{~cm}^{-1} ; D_{x}=1.162 \mathrm{~g} \mathrm{~cm}^{-3}$. Absent reflections: $(h 0 l)$ for $l$ odd, $(0 k 0)$ for $k$ odd. Space group $P 2_{1} / c$ (No. 14).

\section{STRUCTURE DETERMINATIONS}

The structure of tris(morpholino)phosphine was determined from Patterson functions; that of tris(piperidino)phosphine (with two molecules per asymmetric unit) was solved by the use of the program assembly MULTAN. ${ }^{28}$ All positions of the heavy atoms were found after some cycles of successive Fourier syntheses. The refinement proceeded by least-squares methods, initially with isotropic temperature factors, subsequently with anisotropic temperature factors for the heavy atoms. Hydrogen positions were calculated from stereochemical considerations and refined; they were assigned isotropic temperature factors which were refined with the constraint that all hydrogen atoms with about the same distance from the central phosphorus atom were to have the same $B$-value. The refinements converged to conventional $R$ - factors of 0.063 (I) and 0.072 (II); the $R_{w}$-values were 0.069 (I) and 0.092 (II) and the standard deviation of an observation of unit weight, $\left[\Sigma w \Delta F^{2} /(m-n)\right]^{\frac{1}{2}}$, was 2.1 (I) and 2.5 (II), respectively. The overdetermination ratios were 8.4 (I) and 9.9 (II).

The two molecules in the asymmetric unit of II are nearly related by a pseudo screw axis along the $a$-axis close to $y=\frac{1}{4}, z=\frac{1}{8}$. No correlation larger than 0.5 was found between positional parameters, however. The geometry of the two molecules is nearly identical, there are no significant differences in bond lengths and angles nor in $\mathrm{P}-\mathrm{N}$ torsional angles.

Final atomic parameters are listed in Table 1. Tables of observed and calculated structure factors with standard deviations are available from the authors.

ORTEP drawings of molecule I and one of the molecules II are shown in Fig. 2, where the numbering of the atoms is also indicated.

\section{RESULTS}

In Table 2 bond lengths, bond angles, torsional angles and other structural data are given. Estimated standard deviations are calculated from the variance-coveriance matrix. In Fig. 3 are shown the Newman projections of the $\mathrm{P}-\mathrm{N}$ bonds. As the nitrogen bonds are nearly coplanar for some of the nitrogen atoms, the definition of the torsional angle about the $\mathrm{P}-\mathrm{N}$ bond given by Holywell ${ }^{10}$ cannot be applied. According to the IUPAC recommendations ${ }^{29}$ the lone pair directions on the phosphorus and nitrogen atoms decide the description of the conformation. In the present case where neither of the atoms exhibit three-fold symmetry, these direc-
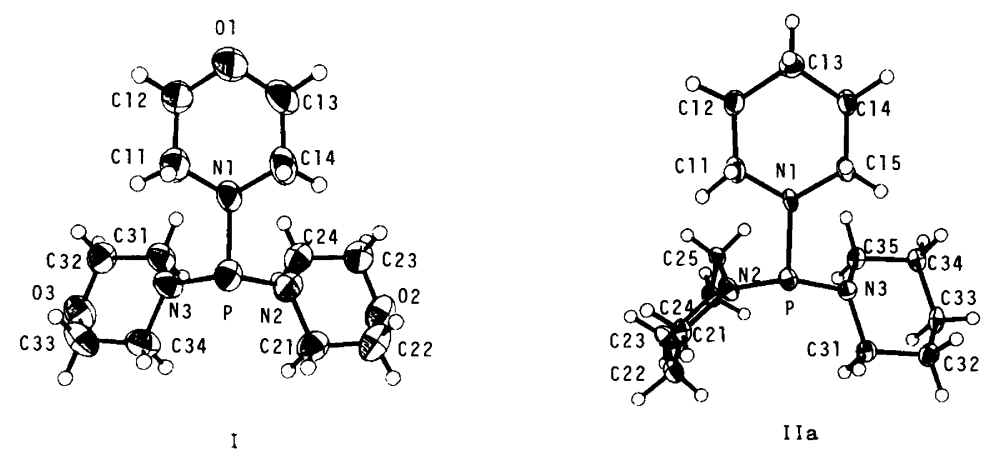

Fig. 2. ORTEP drawings of tris(morpholino)phosphine (I) and tris(piperidino)phosphine (IIa). The atoms of the piperidino substituents of the second molecule of II (IIb) have first index by 3 larger.

Acta Chem. Scand. A 32 (1978) No. 8 
Table 1. Fractional atomic coordinates and thermal parameters with estimated standard deviations for tris(morpholino)phosphine (I) and tris(piperidino)phosphine. The anisotropic temperature factor is given by $\exp -2 \pi^{2}\left(U_{11} a^{* 2} h^{2}+\ldots+2 U_{12} a^{*} b^{*} h k+\ldots\right)$.

I

\begin{tabular}{|c|c|c|}
\hline АTOM & $x$ & $\mathbf{Y}$ \\
\hline $\mathbf{p}$ & .3703111 & .1252111 \\
\hline 01 & .7019131 & $.03011(4)$ \\
\hline .2 & $.2861(4)$ & $.677813 i$ \\
\hline 03 & $-.1570(31$ & $-.3953 i 3 i$ \\
\hline NI & .40468121 & .0718131 \\
\hline N2 & .3609161 & .25251 \\
\hline N3 & $.1807 i 31$ & -.07761 \\
\hline CII & $.52571 \equiv$ & $-.0617(5)$ \\
\hline $\mathrm{Clz}$ &.$(1+2(5)$ & -.1150161 \\
\hline C13 & .7529151 & .1605161 \\
\hline C24 & $.0895(6)$ & .2218151 \\
\hline $\begin{array}{l}\text { c2l } \\
\text { c22 }\end{array}$ & $\begin{array}{l}28131 \\
.3451(1)\end{array}$ & .3658151 \\
\hline$c 23$ & $.3510(5)$ & .3725 (5) \\
\hline$c 26$ & $.2824(5)$ & .2053151 \\
\hline C3! & . 0829151 & -19691 (4) \\
\hline $\begin{array}{l}\text { C32 } \\
633\end{array}$ & $\begin{array}{l}=.0341(5) \\
=.06071,7\end{array}$ & $\begin{array}{l}=3762(5) \\
-.2029(6)\end{array}$ \\
\hline c 34 & .0551 iा 51 & -01036151 \\
\hline
\end{tabular}

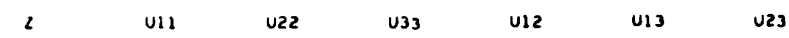

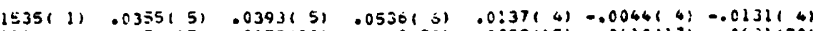
$.311614) \quad .0451(15) \quad .0853(20) \quad 01449(28) \quad .0392115)-.0410117) \quad-.0521(20)$ $.322513) .0596(17) .0560(15) \quad .0748(16) \quad .0376(13) \quad-.0656(1) 1) \quad-.0194(13)$ $.2848(3) .0443(14) .0608(16) \quad .1040124) \quad .099(12) \quad-.0278(15) \quad-.0326(16)$

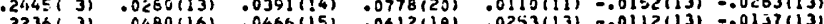

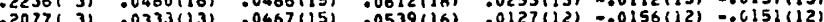

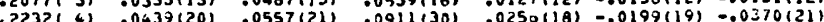
$02176(5) .0526(22): 0061(24): 1250(40) .036 \div(20)=0306(25)=0456(26)$ .3295 ( 5) $: 0409(211): 0783(28) \quad: 0170(33) \quad: 0275(21)=.0345(23) \quad-.0538(271$

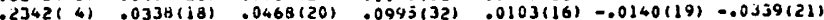

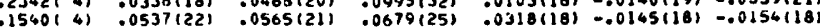
$.1888(4) \quad 0.0757(23) \quad 0.0525(22) \quad: 0746(28) \quad 00368: 22) \quad-.0043(22) \quad-0132(10)$ $.3878(4) \quad .0451(21) \quad .0581(21) \quad .0711(25) \quad .0272(19) \quad-.0046(18) \quad-.0249(19)$ $.3610(3) \quad .0537(22) \quad .0485(19) \quad .0592(22) \quad .0271117)-.0085(17) \quad-.0144(16)$ $.343113) .0509(18) \quad .0493119) \quad .0581(211) .0135(15) \quad-.0126(16) \quad-.0171(16)$

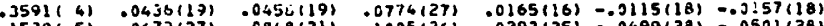

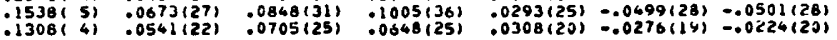

Ni11? $\quad .555(6) \quad-.017(5)$

N!2: $\quad .638161 \quad-.201161$

HI3I

$\begin{array}{ll}.8731 & 01 \\ .7671 & 01\end{array}$

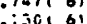

$.258(6)$

$.285(5)$

$.599(6)$

:3:3, b1

$.354(6)$

0.83161

$\begin{aligned}-01 \rightarrow 9(5) & 51 \\ -0309( & 01\end{aligned}$

$-.449(6)$

$7: 05171$

$=.307(6)$

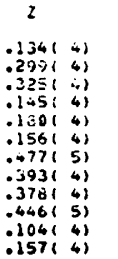

$\begin{array}{cc}8 & \text { ATOM } \\ 4.8121 & 4112 \\ 5.6131 & 1122 \\ 5.6 & 7132 \\ 4.8 & 1142 \\ 4.8 & 11212 \\ 5.6 & 72.22 \\ 5.5 & 7232 \\ 4.8 & 4242 \\ 4.8 & 1312 \\ 5.6 & 1322 \\ 5.6 & 7332 \\ 4.8 & 1342\end{array}$

\begin{tabular}{|c|c|}
\hline & \\
\hline 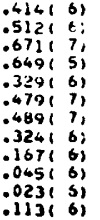 & 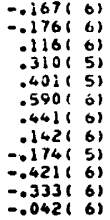 \\
\hline
\end{tabular}

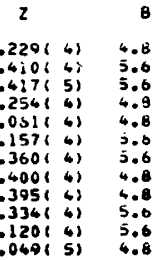

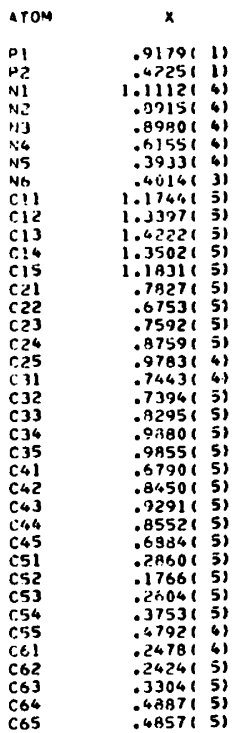

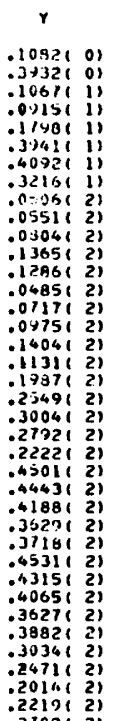

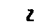

.0402111 . .03672121 .0271 (2) $.2156(2)$ .1102121 .22961 (2) .0615131 .0732131 .0017131

$-.0404121$ .1706121 .2395 ( 3) .3156131 .2854121 .0113121 .00316121
.0101121 .0267121 .0755121 .07571 (19) - 1755131 .2533131 .2809121 .085412 .0167131 -.0612131
-.0323121 .03231 21 .037. .03641 2) .0251 .022 $.2936(3) .0306$ $\begin{array}{ll}.2453(2) & .0352 \\ .2289(2) & .0341 \\ .1804(2) & .0274\end{array}$
U11

.0501610 $.0247(6)$
$.0274(19)$ $.0296(20)$ $.0212(19)$ $.0293(20)$ .0zod (18) $.0353(25)$ $.0291(24)$ $.0282(24)$ $.0296(25)$ $.0293(26)$ $.0291(25)$ $.0232(22)$ $.0297(26)$ $.0422(26)$ $.0312(25)$
$.0287(24)$ .0301 .0341 .0 . .0283

.0319 8319 8 .0438 .0231

.0197
.0212 .02271 .0306

U22

.019455 .0200151 .0203(j) .0205(12) $.0192(16)$
$.0256(1)$ $.0201(16)$ $.0143(22)$
$.02511(22)$ $.0292(24)$ $.0243(23)$ $.0170(20)$ $.0237(25)$ .0365 (30) 02661221 $.0258(22)$ $.0207(22)$ $.0206(21)$ .0231 .0209 .0258

0285

2

1

74

\begin{tabular}{|c|c|c|c|}
\hline U33 & U12 & 013 & 023 \\
\hline 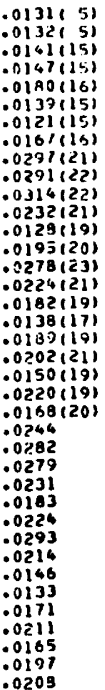 & 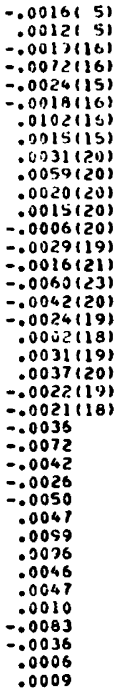 & 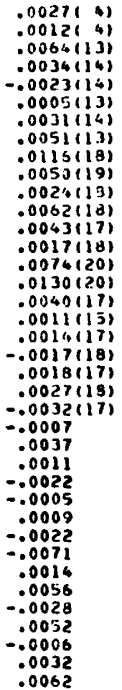 & 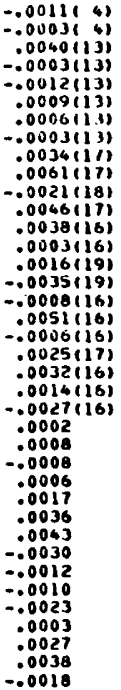 \\
\hline
\end{tabular}


Table 1. Continued.

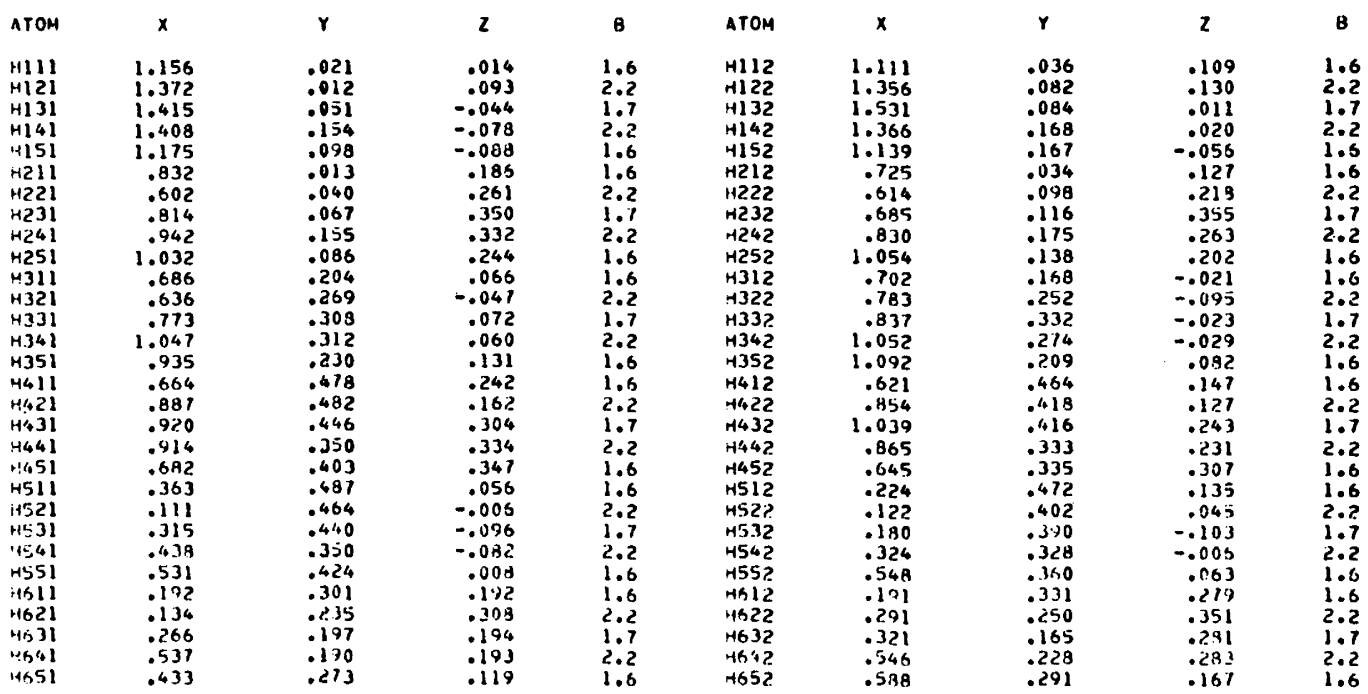

tions are not precisely defined. We have chosen to define the torsional angle as the angle between the lines bisecting the larger of the angles between the projection along the $\mathrm{P}-\mathrm{N}$ bond of the other $\mathrm{P}-\mathrm{N}$ bonds and the $\mathrm{N}-\mathrm{C}$ bonds. The lines thus defining the torsional angles are indicated with broken lines in Fig. 3. It may be noted, however, that the directions thus defined for the phosphorus lone pair direction do not necessarily coincide exactly for the three $\mathrm{P}-\mathrm{N}$ torsional angles of one molecule.
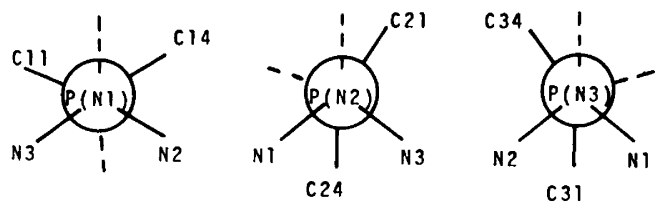

I
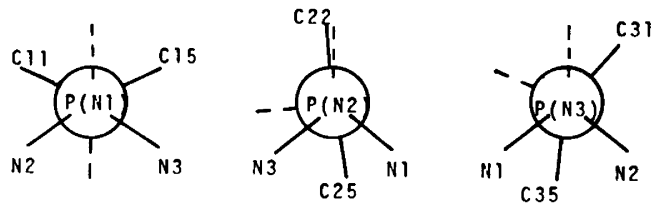

II

Fig. 3. Newman projections along the $\mathrm{P}-\mathrm{N}$ bonds in tris(morpholino)phosphine (I) and tris(piperidinophosphine (II).
Several features in the two structures are similar: Both molecules have two short $\mathrm{P}-\mathrm{N}$ bonds $(1.69-1.70 \AA)$; the third $\mathrm{P}-\mathrm{N}$ bond being significantly longer $(1.726 \AA)$. The nitrogen atoms involved in the long $\mathrm{P}-\mathrm{N}$ bond are essentially $s p^{3}$ hybridized and the lone pair of this nitrogen atom in both compounds is anti to the phosphorus lone pair. The remaining nitrogen atoms are mainly $s p^{2}$ hybridized and their lone pairs are partly orthogonal to that of the phosphorus atom. Two of the $\mathrm{N}-\mathrm{P}-\mathrm{N}$ angles are about $98^{\circ}$ while the third $\mathrm{N}-\mathrm{P}-\mathrm{N}$ angle in both I and II (between bonds to $s p^{2}$ hybridized nitrogen atoms) is about $110^{\circ}$. The sum of the $\mathrm{N}-\mathrm{P}-\mathrm{N}$ bond angles in I and II are 306.6 and $305.0^{\circ}$, respectively.

The significant differences in the two structures are as follows: In II, one of the nitrogen atoms is $s p^{2}$ hybridized, whereas in I all three nitrogen atoms have some $s p^{3}$ character. Furthermore, in I, the two morpholino groups linked through short bonds to the phosphorus atom are twisted in opposite directions (torsional angles of opposite signs), while in II the corresponding piperidino groups are twisted in the same direction.

The morpholino and piperidino parts of the molecule are both in the expected chair conformation. The hybridization of the nitrogen atoms influences slightly the bond angles and bond lengths in these group ( $c f$. Table 3 ). All $\mathrm{N}-\mathrm{P}$ bond directions correspond approximately to that of an 
Table 2. Structural data for tris(morpholino)phosphine (I) and the two crystallographically nonequivalent molecules ( $\mathrm{a}$ and $\mathrm{b}$ ) of tris(piperidino)phosphine (II).

Bond lengths (A)

$\begin{array}{lll} & & I \\ P & \mathrm{~N} 1 & 1.726(3) \\ \mathrm{P} & \mathrm{N} 2 & 1.691(3) \\ \mathrm{P} & \mathrm{N} 3 & 1.696(3) \\ \mathrm{N} 1 & \mathrm{C} 11 & 1.467(4) \\ \mathrm{C} 11 & \mathrm{C} 12 & 1.493(6) \\ \mathrm{C} 12 & \mathrm{O} 1 & 1.431(5) \\ \mathrm{O} 1 & \mathrm{C} 13 & 1.410(5) \\ \mathrm{C} 13 & \mathrm{C} 14 & 1.503(6) \\ \mathrm{C} 14 & \mathrm{~N} 1 & 1.472(4) \\ \mathrm{N} 2 & \mathrm{C} 21 & 1.468(5) \\ \mathrm{C} 21 & \mathrm{C} 22 & 1.498(6) \\ \mathrm{C} 22 & \mathrm{O} 2 & 1.416(5) \\ \mathrm{O} 2 & \mathrm{C} 23 & 1.429(5) \\ \mathrm{C} 23 & \mathrm{C} 24 & 1.495(5) \\ \mathrm{C} 24 & \mathrm{~N} 2 & 1.458(5) \\ \mathrm{N} 3 & \mathrm{C} 31 & 1.464(5) \\ \mathrm{C} 31 & \mathrm{C} 32 & 1.508(5) \\ \mathrm{C} 32 & \mathrm{O} 3 & 1.419(5) \\ \mathrm{O} 3 & \mathrm{C} 33 & 1.414(6) \\ \mathrm{C} 33 & \mathrm{C} 34 & 1.503(6) \\ \mathrm{C} 34 & \mathrm{~N} 3 & 1.455(5) \\ & & \end{array}$

P1
P1
P1
N1
C11
C12
C13
C14
C15
N2
C21
C22
C23
C24
C25
N 3
C 31
C 32
C 33
C 34
C 35

\begin{tabular}{lll} 
& \multicolumn{1}{c}{ IIb } \\
P2 & N4 & $1.724(3)$ \\
P2 & N5 & $1.689(3)$ \\
P2 & N6 & $1.708(3)$ \\
N4 & C41 & $1.472(5)$ \\
C41 & C42 & $1.521(6)$ \\
C42 & C43 & $1.533(6)$ \\
C43 & C44 & $1.532(6)$ \\
C44 & C45 & $1.526(6)$ \\
C45 & N4 & $1.488(5)$ \\
N5 & C51 & $1.457(5)$ \\
C51 & C52 & $1.523(6)$ \\
C52 & C53 & $1.538(6)$ \\
C53 & C54 & $1.519(6)$ \\
C54 54 & C55 & $1.526(6)$ \\
C55 & N5 & $1.464(4)$ \\
N6 & C61 & $1.459(5)$ \\
C61 & C62 & $1.519(6)$ \\
C62 & C63 & $1.528(6)$ \\
C63 & C64 & $1.517(6)$ \\
C64 & C65 & $1.534(5)$ \\
C65 & N6 & $1.469(5)$
\end{tabular}

$\begin{array}{lllr}\text { Bond ang1es } & \left({ }^{\circ}\right) \\ & & \mathrm{I} \\ \mathrm{N} 1 & \mathrm{P} & \mathrm{N} 2 & 98.0(1) \\ \mathrm{N} 2 & \mathrm{P} & \mathrm{N} 3 & 110.7(1) \\ \mathrm{N} 3 & \mathrm{P} & \mathrm{N} 1 & 97.9(1) \\ \mathrm{P} & \mathrm{N} 1 & \mathrm{C} 11 & 114.4(2) \\ \mathrm{P} & \mathrm{N} 1 & \mathrm{C} 14 & 115.0(2) \\ \mathrm{P} & \mathrm{N} 2 & \mathrm{C} 21 & 116.7(2) \\ \mathrm{P} & \mathrm{N} 2 & \mathrm{C} 24 & 125.9(2) \\ \mathrm{P} & \mathrm{N} 3 & \mathrm{C} 31 & 124.3(2) \\ \mathrm{P} & \mathrm{N} 3 & \mathrm{C} 34 & 115.6(2) \\ \mathrm{N} 1 & \mathrm{C} 11 & \mathrm{C} 12 & 110.2(3) \\ \mathrm{C} 11 & \mathrm{C} 12 & \mathrm{O} 1 & 112.7(4) \\ \mathrm{C} 12 & \mathrm{O} 1 & \mathrm{C} 13 & 109.4(3) \\ \mathrm{O} 1 & \mathrm{C} 13 & \mathrm{C} 14 & 112.8(4) \\ \mathrm{C} 13 & \mathrm{C} 14 & \mathrm{~N} 1 & 109.6(3) \\ \mathrm{C} 14 & \mathrm{~N} 1 & \mathrm{C} 11 & 108.2(3) \\ \mathrm{N} 2 & \mathrm{C} 21 & \mathrm{C} 22 & 109.8(3) \\ \mathrm{C} 21 & \mathrm{C} 22 & \mathrm{O} 2 & 111.9(3) \\ \mathrm{C} 22 & \mathrm{O} 2 & \mathrm{C} 23 & 109.7(3) \\ \mathrm{O} 2 & \mathrm{C} 23 & \mathrm{C} 24 & 110.9(3) \\ \mathrm{C} 23 & \mathrm{C} 24 & \mathrm{~N} 2 & 109.7(3) \\ \mathrm{C} 24 & \mathrm{~N} 2 & \mathrm{C} 21 & 110.6(3) \\ \mathrm{N} 3 & \mathrm{C} 31 & \mathrm{C} 32 & 109.8(3) \\ \mathrm{C} 13 & \mathrm{C} 32 & \mathrm{O} 3 & 111.0(3) \\ \mathrm{C} 32 & \mathrm{O} 3 & \mathrm{C} 33 & 110.0(3) \\ \text { O3 } & \mathrm{C} 33 & \mathrm{C} 34 & 111.5(4) \\ \mathrm{C} 33 & \mathrm{C} 34 & \mathrm{~N} 3 & 109.8(3) \\ \mathrm{C} 34 & \mathrm{~N} 3 & \mathrm{C} 31 & 110.6(3) \\ & & & \end{array}$

$\begin{array}{ll}N 1 & P 1 \\ N 2 & P 1 \\ N 3 & P 1 \\ P 1 & N 1 \\ P 1 & N 1 \\ P 1 & N 2 \\ P 1 & N 2 \\ P 1 & N 3 \\ \text { P1 } & N 3 \\ N 1 & C 11 \\ C 11 & C 12 \\ C 12 & C 13 \\ C 13 & C 14 \\ C 14 & C 15 \\ C 15 & N 1 \\ N 2 & C 21 \\ C 21 & C 22 \\ C 22 & C 23 \\ C 23 & C 24 \\ C 24 & C 25 \\ C 25 & N 2 \\ N 3 & C 31 \\ C 31 & C 32 \\ C 32 & C 33 \\ C 33 & C 34 \\ C 34 & C 35 \\ C 35 & N 3 \\ & \end{array}$

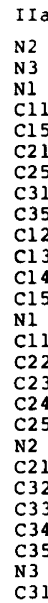

$$
\begin{array}{r}
98.9(2) \\
109.2(2) \\
97.0(2) \\
113.3(3) \\
116.4(3) \\
121.0(3) \\
126.0(3) \\
114.6(3) \\
123.7(3) \\
110.7(3) \\
111.3(3) \\
110.5(3) \\
110.3(3) \\
110.7(3) \\
109.9(3) \\
111.0(3) \\
111.7(3) \\
110.9(3) \\
110.0(3) \\
110.6(3) \\
112.9(3) \\
111.7(3) \\
110.9(3) \\
109.8(3) \\
110.5(3) \\
110.6(3) \\
112.1(3)
\end{array}
$$

$\begin{array}{lllr} & & \text { IIb } & \\ \text { N4 } & \text { P2 } & \text { N5 } & 98.8(2) \\ \text { N5 } & \text { P2 } & \text { N6 } & 108.9(2) \\ \text { N6 } & \text { P2 } & \text { N4 } & 97.0(2) \\ \text { P2 } & \text { N4 } & \text { C41 } & 113.4(3) \\ \text { P2 } & \text { N4 } & \text { C45 } & 115.7(2) \\ \text { P2 } & \text { N5 } & \text { C51 } & 120.5(2) \\ \text { P2 } & \text { N5 } & \text { C55 } & 126.6(3) \\ \text { P2 } & \text { N6 } & \text { C61 } & 114.5(3) \\ \text { P2 } & \text { N6 } & \text { C65 } & 123.5(2) \\ \text { N4 } & \text { C41 } & \text { C42 } & 110.1(3) \\ \text { C41 } & \text { C42 } & \text { C43 } & 110.8(3) \\ \text { C42 } & \text { C43 } & \text { C44 } & 110.1(3) \\ \text { C43 } & \text { C-4 } & \text { C45 } & 110.6(3) \\ \text { C44 } & \text { C45 } & \text { N4 } & 109.7(3) \\ \text { C45 } & \text { N4 } & \text { C41 } & 109.8(3) \\ \text { N5 } & \text { C51 } & \text { C52 } & 111.3(3) \\ \text { C51 } & \text { C52 } & \text { C53 } & 111.0(3) \\ \text { C52 } & \text { C53 } & \text { C54 } & 111.2(3) \\ \text { C53 } & \text { C54 } & \text { C55 } & 110.1(3) \\ \text { C54 } & \text { C55 } & \text { N5 } & 110.7(3) \\ \text { C55 } & \text { N5 } & \text { C51 } & 112.5(3) \\ \text { N6 } & \text { C61 } & \text { C62 } & 111.8(3) \\ \text { C61 } & \text { C62 } & \text { C63 } & 110.9(3) \\ \text { C62 } & \text { C63 } & \text { C64 } & 109.9(3) \\ \text { C63 } & \text { C64 } & \text { C65 } & 110.3(3) \\ \text { C64 } & \text { C65 } & \text { N6 } & 110.6(3) \\ \text { C65 } & \text { N6 } & \text { C61 } & 111.9(3)\end{array}$

Torsional angles $\left({ }^{\circ}\right)$

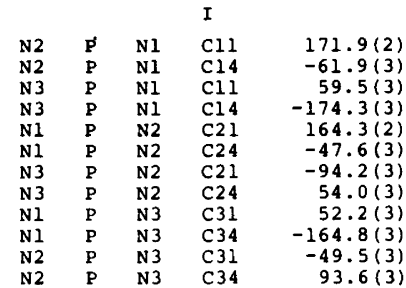

\begin{tabular}{llllr} 
II & \multicolumn{3}{r}{ (average } & for $a$ and $b$ ) \\
N2 & P1 & N1 & C11 & $61.5(3)$ \\
N2 & P1 & N1 & C15 & $-170.0(3)$ \\
N3 & P1 & N1 & C11 & $172.1(2)$ \\
N3 & P1 & N1 & C15 & $-59.4(3)$ \\
N1 & P1 & N2 & C21 & $132.6(3)$ \\
N1 & P1 & N2 & C25 & $40.6(3)$ \\
N3 & P1 & N2 & C21 & $126.7(3)$ \\
N3 & P1 & N2 & C25 & $-60.0(3)$ \\
N1 & P1 & N3 & C31 & $171.1(2)$ \\
N1 & P1 & N3 & C35 & $-46.1(3)$ \\
N2 & P1 & N3 & C31 & $-87.1(3)$ \\
N2 & P1 & N3 & C35 & $55.8(3)$
\end{tabular}


Table 2. Continued.

Various derived and averaged data

\begin{tabular}{|c|c|c|c|c|c|c|}
\hline \multirow[b]{2}{*}{ Nx } & \multicolumn{3}{|c|}{ I } & \multicolumn{3}{|c|}{ II (average for $a$ and $b$ ) } \\
\hline & N1 & $\mathrm{N} 2$ & N3 & N1 & N2 & N3 \\
\hline $\begin{array}{l}\overline{N-C}(A) \\
\frac{A-C}{C-O}(A) \\
\text { Deviation of } \mathrm{Nx}\end{array}$ & $\begin{array}{l}1.470 \\
1.498 \\
1.421\end{array}$ & $\begin{array}{l}1.463 \\
1.497 \\
1.423\end{array}$ & $\begin{array}{l}1.460 \\
1.505 \\
1.417\end{array}$ & $\begin{array}{l}1.478 \\
1.524\end{array}$ & $\begin{array}{l}1.459 \\
1.526\end{array}$ & $\begin{array}{l}1.464 \\
1.526\end{array}$ \\
\hline $\begin{array}{l}\text { from plane PCC }(\AA) \\
\text { Sum of } N \text { bond angles }\left({ }^{\circ}\right) \\
\text { Torsional angle }\end{array}$ & $\begin{array}{r}0.43 \\
337.6\end{array}$ & $\begin{array}{r}0.23 \\
353.2\end{array}$ & $\begin{array}{r}0.27 \\
350.5\end{array}$ & $\begin{array}{r}0.42 \\
339.3\end{array}$ & $\begin{array}{r}0.05 \\
359.8\end{array}$ & $\begin{array}{r}0.29 \\
350.1\end{array}$ \\
\hline Lone pair $(\mathrm{P})-\mathrm{P}-\mathrm{N}$-Lone pair $(\mathrm{N})\left({ }^{\circ}\right)$ & 170.6 & -70.9 & 73.2 & -178.9 & -96.3 & -66.6 \\
\hline $\begin{array}{l}\overline{\mathrm{C}-\mathrm{H}}(\mathrm{A}) \\
\text { Sum of } \mathrm{P} \text { bond angles }\left({ }^{\circ}\right)\end{array}$ & & $\begin{array}{l}0.99(6) \\
306.6\end{array}$ & & & $\begin{array}{l}1.00(5) \\
305.0\end{array}$ & \\
\hline
\end{tabular}

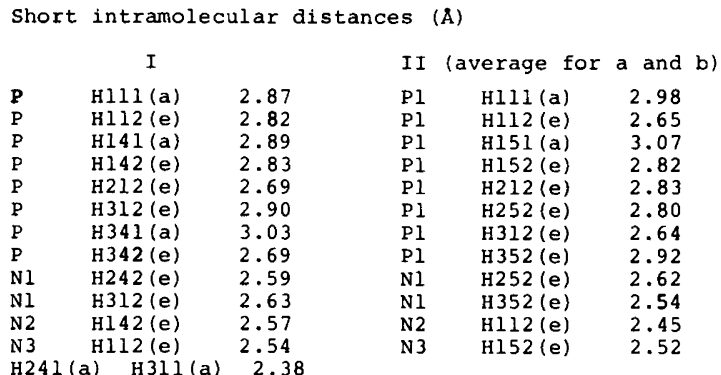

equatorial bond of the morpholine and piperidine rings.

In both I and II the packing of the molecules in the crystals is of normal van der Waals' type, the contacts being mainly between hydrogen atoms.

\section{DISCUSSION}

Conformational considerations. Recent NMR studies of acyclic amino phosphines of the general type $\mathrm{X}_{2} \mathrm{PNR}_{2}(\mathrm{X}=\mathrm{F}$ and $\mathrm{Cl})$ and related substances have revealed that the synclinal (gauche) conformation is the best representation of the ground state geometry, regardless whether the nitrogen atom is $s p^{2}$ or $s p^{3}$ hybridized. ${ }^{30}$ The preference for this conformation is generally thought to be due to the lone pair repulsion which is at its minimum in the synclinal conformation. This orthogonality of lone-pair electrons appears to be quite general and is apparent in compounds with $\mathrm{S}-\mathrm{S},{ }^{31} \mathrm{~N}-\mathrm{N},{ }^{32}$ $\mathrm{N}-\mathrm{S},{ }^{33}$ and $\mathrm{O}-\mathrm{O}^{34}$ bonds. For a detailed discussion of the "gauche" effect, $c f$. Ref. 35.

However, when the phosphorus atom is bonded to three atoms, each with a lone pair as in tris(dialkylamino) phosphines, the situation is more complex. Cowley et al. ${ }^{1}$ have argued convincingly in favour of a conformation where only two nitrogen lone pairs are orthogonal to the phosphorus lone pair. The results from the present study, however, suggest that the steric demands of the substituents linked to the nitrogen atoms must be taken into account when the conformation of tris(dialkylamino)phosphines is to be considered. Evidently, a complex interplay of effects due to lone pair repulsions, phosphorus-nitrogen bond lengths, hybridization of nitrogen atoms, bond angles around the phosphorus atom and the spatial requirements of the substituents determine the geometry.

For the discussion of the lone pair directions the same difficulty arises as when defining the torsional angles. We take as the direction of the lone pair on a trivalent atom that of the normal to the plane through three points in unit distance from the atom along the bonds. In Table 3 are listed angles between such directions in the present molecules.

In both I and II, two of the nitrogen lone pairs are partly orthogonal to the phosphorus lone pair in agreement with the suggestion by Cowley. ${ }^{1}$ The third lone pair is nearly antiparallel to that of the phosphorus atom, all nitrogen lone pairs are thus 
Table 3. Angles $\left({ }^{\circ}\right)$ between lone pair directions of the $\mathrm{P}$ and $\mathrm{N}$ atoms of $\mathrm{I}$ and the two molecules (a and b) of II (for definition, see text).

\begin{tabular}{|c|c|c|c|}
\hline Atoms & Molecule I & Molecule IIa & Molecule IIb \\
\hline $\mathrm{P} \quad \mathrm{N} 1$ & 170.6 & 169.4 & 169.6 \\
\hline$P \quad \mathrm{~N} 2$ & 70.9 & 79.8 & 79.4 \\
\hline $\mathrm{P} \quad \mathrm{N} 3$ & 73.2 & 68.3 & 68.8 \\
\hline N1 N2 & 114.9 & 71.0 & 71.0 \\
\hline N2 N3 & 83.4 & 74.2 & 73.7 \\
\hline N1 N3 & 109.1 & 119.1 & 118.8 \\
\hline
\end{tabular}

also partly orthogonal to each other. According to the figures given in Table 3 none of the structures proposed in Fig. 1 are representative of the structure of I and II in their crystalline state. Because of the close similarity of the structures of I and II, and because the spatial demands of the usual dialkylamino groups are quite comparable to those of the piperidino and the morpholino groups, one may conclude that tris(dialkylamino)phosphines $\left(\mathrm{R}_{2} \mathrm{~N}\right)_{3}(\mathrm{R}=\mathrm{Me}$, Et etc.), generally have geometries of the same type as have I and II. The close similarity in the structures of tris(morpholino)phosphine selenide, tris(piperidino)phosphine selenide and tris(dimethylamino)phosphine selenide ${ }^{36}$ substantiates this conclusion.

The important factor which is primarily responsible for the geometry of tris(dialkylamino)phosphines appears thus to be the accommodation of two, but only two, nitrogen atoms in positions whereby their lone pair directions are close to be orthogonal to the phosphorus lone pair. ${ }^{1}$ The third substituent will then be accommodated where vacant space is available. It is noteworthy that the structures of I and II bear no resemblance to that of triaryl phosphines, $\operatorname{Ar}_{3} \mathrm{P}$, where the aryl groups are twisted to propeller-shaped molecules. ${ }^{37} \mathrm{Ap}$ parently the repulsive forces between lone pairs will not allow geometries of this type for tris(dialkylamino)phosphines.

Bond angles around the phosphorus atom. The sum of the bond angles, $\Sigma \angle$ NPN, in I and II are 306.6 and $305.0^{\circ}$, respectively, which is approximately the same as in $\mathrm{Ar}_{3} \mathrm{P}^{37}{ }^{37}$ Except for $\mathrm{PH}_{3}$ and the sterically very hindered phosphine, tris-tert-butyl phosphine, ${ }^{38}$ the sum of bond angles around the phosphorus atom in tervalent phosphorus compounds is generally in the range $295-310^{\circ}$ and is not very dependent on the substituents. It is an interesting observation in the present compounds that one of the NPN bond angles, the angle between bonds to the essentially $s p^{2}$ hybridized nitrogen atoms, is considerably larger $\left(110^{\circ}\right)$ than the two remaining NPN bond angles $\left(98^{\circ}\right)$. This increase in the one bond angle is probably due to the steric demands of the two substituents whose nitrogen atoms have their lone pair orthogonal to the phosphorus lone pair.

Bond angles around the nitrogen atoms. In both I and II there are actually three different nitrogen atoms, the sum of their bond angles being 337.6, 353.2 and $350.2^{\circ}$ in $I$ and $339.3,359.8$ and $350.1^{\circ}$ in II. The nitrogen atom with its lone pair anti to the phosphorus lone pair is essentially $s p^{3}$ hybridized in both I and II as seen from the sum of the bond angles, 337.6 and $339.3^{\circ}$, respectively. It may well be that when no significant "through-space" $\sigma$ or $\pi$ interaction with the phosphorus lone pair takes place due to the unsuitable spatial orientation of the nitrogen lone pair, the $s p^{3}$ state is the favourable hybridization of the nitrogen atom. ${ }^{23}$ Furthermore. increased $p$-character of the nitrogen atom is accompanied by a significant lengthening of the phosphorus-nitrogen bond, which may be of some advantage when the substituent is to be accommondated on the fairly crowded central phosphorus atom.

The phosphorus-nitrogen bond lengths. In recent years a number of X-ray, electron diffraction and microwave studies of compounds with phosphorusnitrogen bonds have appeared and the $\mathrm{P}-\mathrm{N}$ bond is known to range from 1.84 to $1.47 \AA$ (cf. Refs. 13 and 39 for a survey of $\mathrm{P}-\mathrm{N}$ bond lengths). It is generally accepted that $\mathbf{P}^{\mathrm{v}}-\mathrm{N}$ bond lengths shorter than $1.77 \AA$ and $\mathrm{P}^{\mathrm{III}}-\mathrm{N}$ bond lengths shorter than $1.80 \AA$ reflect bond orders higher than unity. ${ }^{40-42}$ Bond orders higher than unity seem invariably to be connected with some deviation from $s p^{3}$ hybridization of the nitrogen atom.

In both I and II the two shorter bonds (1.69-1.70 $\AA$ ), and the longer bond (1.726 $\AA$ ), are considerably shorter than the length for a single $\mathrm{P}-\mathrm{N}$ bond according to Pauling $(1.80 \AA),{ }^{40}$ and also shorter than the Schomaker-Stevenson value $(1.76 \AA) .{ }^{42}$ In Fig. 4 is plotted the sum of the nitrogen bond angles versus the corresponding $\mathrm{P}-\mathrm{N}$ bond lengths in I and II. There seems to be a linear dependence, and the least-squares straight line is indicated. From extrapolation to an angle sum of $325-330^{\circ}$ a $\mathrm{P}^{\mathrm{III}}-\mathrm{N}_{s p^{3}}$ bond length of $1.74-1.75 \AA$ may be obtained as the measure for an authentic $\mathrm{P}^{\mathrm{III}}-\mathrm{N}$ single bond. This is slightly shorter than the 


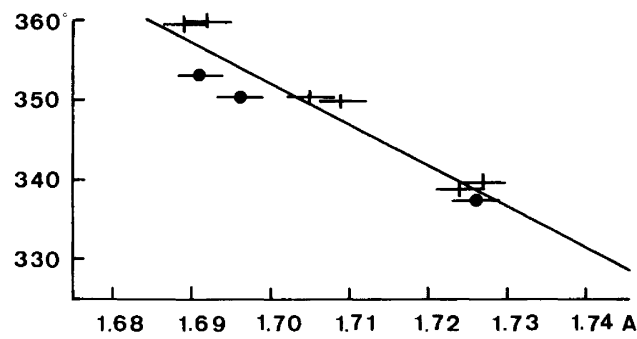

Fig. 4. The $\mathrm{P}-\mathrm{N}$ bond length $v$ s. the nitrogen bond angle sum in tris(morpholino)phosphine (circles) and tris(piperidino)phosphine (crosses).

current estimate of a $\mathrm{P}-\mathrm{N}$ single bond, $1.77 \AA$, the bond length in the monoamidophosphate anion, ${ }^{41}$ which, however, exists in the betaine form.

IR studies of tris(dimethylamino)phosphine and tris(pyrrolidino)phosphine have shown two peaks in the $\mathrm{P}-\mathrm{N}$ stretching region which have been assigned to symmetric and asymmetric stretching. ${ }^{14,43}$ In view of the present results indicating that in this class of compounds there are at least two different $\mathrm{P}-\mathrm{N}$ bonds, the IR assignments ought to be reconsidered.

The torsional angles. Apart from the torsional angle of the $\mathrm{P}-\mathrm{N}$ bonds for the essentially $s p^{3}$ hybridized nitrogen atoms which are about $180^{\circ}$, the remaining angles are in the range $69-96^{\circ}$, most of them around $70^{\circ}$ (Table 2). None of the lone pairs of the nitrogen atoms are ideally orthogonal to the phosphorus lone pair. It appears that the value of $70^{\circ}$ for the torsional angle is the optimum compromise between steric repulsion of the atomic cores and the electronic energy. This value has previously been found in tris(dimethylamino)difluorophosphorane. ${ }^{44}$ However, the $\mathrm{P}-\mathrm{N} 2$ torsional angle of II of $96^{\circ}$ is associated with the shortest (strongest) $\mathrm{P}-\mathrm{N}$ bond and with the most coplanar nitrogen bonds of all in the present analysis; furthermore, this N2 lone pair is also the one closest to being orthogonal to the phosphorus lone pair $\left(80^{\circ}\right)$.

The structure of the substituents. The morpholino and piperidino substituents are all in the expected chair conformation and linked to the phosphorus atom in an approximately equatorial direction. As indicated in Table 2, the bond angles and, to some extent the bond lengths, are significantly dependent upon the hybridization of the nitrogen atoms. A detailed discussion of bond lengths and angles in the morpholino and the piperidino group will appear in a later paper in this series.

Intramolecular contacts. Several short intramolecular nonbonded contacts have been observed and are listed in Table 2.

In compound I the four hydrogen atoms of $\mathrm{C} 11$ and $\mathrm{C} 14$ are about equidistant from $\mathrm{P}$ [2.82-2.89 $\AA]$; the equatorial hydrogen atoms of $\mathrm{C} 11$ and $\mathrm{C} 14$ are also in van der Waals' contact with $\mathrm{N} 3$ and N2, respectively [ 2.54 and $2.57 \AA]$. The equatorial hydrogen atoms of $\mathrm{C} 24$ and $\mathrm{C} 31$ are both at van der Waals' distance from N1 [2.59 and $2.63 \AA$, respectively], and the axial hydrogen atoms of the same carbon atoms are in contact with each other $[2.38 \AA]$. Probably these interactions are responsible for the asymmetry in the external angles at $\mathrm{N} 2$ and N3 $\left[\angle \mathrm{P}-\mathrm{N} 2-\mathrm{C} 21=116.7^{\circ}, \quad \angle \mathrm{P}-\mathrm{N} 2-\mathrm{C} 24=\right.$ $125.9^{\circ} ; \angle \mathrm{P}-\mathrm{N} 3-\mathrm{C} 34=115.6^{\circ}, \angle \mathrm{P}-\mathrm{N} 3-\mathrm{C} 31=$ $\left.124.3^{\circ}\right]$; they are counterbalanced only by the interactions between the equatorial hydrogen atoms at $\mathrm{C} 21$ and $\mathrm{P}[2.69 \AA]$ and $\mathrm{C} 34$ and $\mathrm{P}[2.69 \AA]$. The steric demands of the substituents are also the probable cause for the $\mathrm{N} 2-\mathrm{P}-\mathrm{N} 3$ angle being as large as $110.7^{\circ}$.

The situation is somewhat different in compound II since the $\mathrm{P}-\mathrm{N} 2$ twist is in the opposite direction as compared to the $\mathrm{P}-\mathrm{N} 3$ twist in I (cf. Fig. 2). Since the molecules $a$ and $b$ of II are nearly identical, mean values are used in the discussion. As in I the four hydrogen atoms of C11 and C15 of II are in contact with the phosphorus atom [2.65-3.07 $\AA]$ and the equatorial ones in contact with $\mathrm{N} 2$ and N3 [2.45 and $2.52 \AA$, respectively]. The atoms of the ring associated with $\mathrm{N} 3$ have mainly contacts analogous to those described for compound I [equatorial hydrogen of C35 to N1: $2.54 \AA$, that of C31 to P: $2.64 \AA$ ] and the same asymmetry in the external angles of $\mathrm{N} 3$ is observed $[\angle \mathrm{P}-\mathrm{N} 3-\mathrm{C} 31$ $\left.=114.6^{\circ}, \angle \mathrm{P}-\mathrm{N} 3-\mathrm{C} 35=123.6^{\circ}\right]$. There are no contacts between hydrogens of $\mathrm{C} 35$ and $\mathrm{C} 25$, however. The equatorial hydrogen atom of $\mathrm{C} 25$ is separated from N1 by $2.62 \AA$ and from $\mathrm{P}$ by $2.80 \AA$; the equatorial hydrogen atom of $\mathrm{C} 21$ is at a distance $2.83 \AA$ from $\mathrm{P}$. The asymmetry in the external angles of $\mathrm{N} 2$ is less pronounced than for $\mathrm{N} 3$ $\left[\angle \mathrm{P} 1-\mathrm{N} 2-\mathrm{C} 21=120.8^{\circ}, \quad \angle \mathrm{P} 1-\mathrm{N} 2-\mathrm{C} 25=\right.$ $\left.126.3^{\circ}\right]$; the angle $\mathrm{N} 2-\mathrm{P}-\mathrm{N} 3\left[109.1^{\circ}\right]$ is slightly smaller than in $\mathrm{I}$.

Comments on the reactivity of $I$ and II. From a recent kinetic study of reactions with methyl iodide it has been found that II is considerably more nucleophilic than $\mathrm{I} .{ }^{45}$ However, the similarity in the 
structure of I and II including bond lengths and bond angles does not signal such a difference in reactivity. The presence of one essentially $s p^{3}$ hybridized nitrogen atom in I and II agrees with the fact that tris(dialkylamino)phosphines may act as nitrogen nucleophiles toward several substrates. ${ }^{11.12}$

\section{CONCLUSIONS}

None of the structural models considered for the structure of tris(dialkylamino)phosphines, Fig. 1, are in accordance with the structure of tris(piperidino)phosphine and tris(morpholino)phosphine. In this class of compounds two substituents appear to be accommodated with essentially $s p^{2}$ hybridized nitrogen atoms in positions whereby their lone pairs are partly orthogonal to the phosphorus lone pair. The position of the third substituent appears to be mainly governed by the steric demands. In both I and II the nitrogen atom of this third substituent is essentially $s p^{3}$ hybridized and has its lone pair anti relative to the phosphorus lone pair. The results indicate that in tris(dialkylamino)phosphines there are nitrogen atoms with at least two different hydridizations and thus at least two types of $\mathrm{P}-\mathrm{N}$ bonds in each molecule. In view of this, it appears as if the results from recent IR, Raman and photoelectron spectral studies of tris(dialkylamino)phosphines and related compounds ought to be reconsidered.

\section{REFERENCES}

1. Cowley, A. H., Dewar, M. J. S., Goodman, D. W. and Schweiger, J. R. J. Am. Chem. Soc. 95 (1973) 6506.

2. Lappert, M. F., Pedley, J. B., Wilkins, B. T., Stelzer, O. and Unger, E. J. Chem. Soc. Dalton Trans. (1975) 1207.

3. Cowley, A. H., Goodman, D. W., Knebler, N. A., Sanchez, M. and Verkade, J. G. Inorg. Chem. $16(1977) 854$.

4. Hargis, J. H. and Worley, S. D. Inorg. Chem. 16 (1977) 1886.

5. Cowley, A. H. Phosphorus and Sulphur 2 (1976) 283.

6. Morris, E. D. and Nordman, C. E. Inorg. Chem. 8 (1969) 1673.

7. Vilkov, L. V., Khaikin, L. S. and Evdokimov, V. V. Zh. Strukt. Khim. 13 (1972) 7.

8. Brittain, A. H., Smith, J. E., Lee, P. L., Cohn, K. and Schwendeman, R. H. J. Am. Chem. Soc. 93 (1971) 6772.
9. Forti, P., Damiani, D. and Favero, P. G. J. Am. Chem. Soc. 95 (1973) 756.

10. Holywell, G. C., Rankin, D. W. H., Beagley, B. and Freeman, J. M. J. Chem. Soc. A (1971) 785.

11. Simonnin, M. P., Charrier, C. and Burgada, R. Org. Magn. Reson. 4 (1972) 113.

12. Hudson, R. F. and Brown, C. Acc. Chem. Res. 5 (1972) 204.

13. Clardy, J. C., Kolpa, R. L. and Verkade, J. G. Phosphorus 4 (1974) 133.

14. Mathis, R., Lafaille, L. and Burgada, R. Spectrochim. Acta A 30 (1974) 357.

15. Gilje, J. W. and Seff, K. Inorg. Chem. 11 (1972) 1643.

16. Bach, M. C., Brian, C., Crasnier, F., Labarre, J.-F., Leibovici, C. and Dargelos, A. J. Mol. Struct. 17 (1973) 23.

17. Emsley, J. and Hall, D. The Chemistry of Phosphorus, Harper and Row, London 1976, p. 394.

18. Hoffmann, R., Howell, J. M. and Muetterties, E. L. J. Am. Chem. Soc. 94 (1972) 3047.

19. Strich, A. and Veillard. A. J. Am. Chem. Soc. 95 (1973) 5574.

20. Hoffmann, R. Acc. Chem. Res. 4 (1971) 1.

21. Czismadia, I. G., Cowley, A. H., Taylor, M. W., Tel, L. M. and Wolfe, S. Chem. Commun. (1972) 1147.

22. Czismadia, I. G., Cowley, A. H., Taylor, M. W. and Wolfe, S. Chem. Commun. (1974) 432.

23. Lambert, J. B. Top. Stereochem. 6 (1971) 19.

24. Stangeland, L. J., Austad, T. and Songstad, J. Acta Chem. Scand. 27 (1973) 3919.

25. Groth, P. Acta Chem. Scand. 27 (1973) 1837.

26. Doyle, P. A. and Turner, P. S. Acta Crystallogr. A 24 (1968) 390.

27. Stewart, R. F., Davidson, E. R. and Simpson, W. T. J. Chem. Phys. 42 (1965) 3175.

28. Germain, G., Main, P. and Woolfson, M. M. Acta Crystallogr. A 27 (1971) 368.

29. IUPAC Commission on Nomenclature of Organic Chemistry, Pure Appl. Chem. 45 (1976) 11.

30. Cowley, A. H., Dewar, M. J. S., Jackson, W. R. and Jennings, W. B. J. Am. Chem. Soc. 92 (1970) $1085,5206$.

31. Foss, O. In Kharasch, N., Ed., Organic Sulfur Compounds, Pergamon, New York 1961, Vol. 1, Chapter 8.

32. Dewar, M. J. S. and Jennings, W. B. J. Am. Chem. Soc. 91 (1969) 3656.

33. Raban, M., Kenney, K. W. J., Jr. and Jones, F. B. J. Am. Chem. Soc. 91 (1969) 6677.

34. Fink, W. H. and Allen, L. C. J. Chem. Phys. 46 (1967) 2261.

35. Wolfe, S. Acc. Chem. Res. 5 (1972) 102.

36. Rømming, C. and Songstad, J. Acta Chem. Scand. To be published.

Acta Chem. Scand. A 32 (1978) No. 8 
37. Corbridge, D. E. C. The Structural Chemistry of Phosphorus, Elsevier, Amsterdam 1974, p. 330.

38. Labarre, M.-C. and Hausard, M. J. Mol. Struct. 26 (1975) 17.

39. Vilkov, L. V. and Khaikin, L. S. Top.Curr. Chem. 53 (1975) 25.

40. Pauling, L. Nature of the Chemical Bond, Cornell University Press, New York 1960.

41. Cruickshank, D. W. J. Acta Crystallogr. 17 (1964) 671.

42. Schomaker, V. and Stevenson, D. P. J. Am. Chem. Soc. 63 (1941) 37.

43. Raüchle, F., Pohl, W., Blaich, B. and Goubeau, J. Ber Bunsenges. Phys. Chem. 75 (1971) 66.

44. Oberhammer, H. and Schmutzler, R. J. Chem. Soc. Dalton Trans. (1976) 1454.

45. Thorstenson, T. and Songstad, J. Acta Chem. Scand. A 30 (1976) 781.

Received April 24, 1978. 